University of Nebraska - Lincoln

DigitalCommons@University of Nebraska - Lincoln

\title{
Demonstration of an ultraviolet ZnO-based optically pumped third order distributed feedback laser
}

\author{
Daniel Hofstetter \\ Institute of Physics, University of Neuchatel, 1 A.-L. Breguet, CH-2000 Neuchatel, Switzerland \\ Yargo Bonetti \\ Institute of Physics, University of Neuchatel, 1 A.-L. Breguet, CH-2000 Neuchatel, Switzerland \\ Fabrizio Giorgetta \\ Institute of Physics, University of Neuchatel, 1 A.-L. Breguet, CH-2000 Neuchatel, Switzerland \\ Abdel-Hamid El-Shaer \\ Institute for Semiconductor Technology, Technical University of Braunschweig, Hans-Sommer-Strasse 66, \\ D-38106 Braunschweig, Germany \\ Andrey Bakin \\ Institute for Semiconductor Technology, Technical University of Braunschweig, Hans-Sommer-Strasse 66, \\ D-38106 Braunschweig, Germany \\ See next page for additional authors \\ Follow this and additional works at: https://digitalcommons.unl.edu/mrsecfacpubs \\ Part of the Materials Science and Engineering Commons
}

Hofstetter, Daniel; Bonetti, Yargo; Giorgetta, Fabrizio; El-Shaer, Abdel-Hamid; Bakin, Andrey; Waag, Andreas; Schmidt-Grund, Rudiger; Schubert, Mathias; and Grundmann, Marius, "Demonstration of an ultraviolet ZnO-based optically pumped third order distributed feedback laser" (2007). Faculty Publications: Materials Research Science and Engineering Center. 62.

https://digitalcommons.unl.edu/mrsecfacpubs/62

This Article is brought to you for free and open access by the Materials Research Science and Engineering Center at DigitalCommons@University of Nebraska - Lincoln. It has been accepted for inclusion in Faculty Publications: Materials Research Science and Engineering Center by an authorized administrator of DigitalCommons@University of Nebraska - Lincoln. 


\section{Authors}

Daniel Hofstetter, Yargo Bonetti, Fabrizio Giorgetta, Abdel-Hamid El-Shaer, Andrey Bakin, Andreas Waag, Rudiger Schmidt-Grund, Mathias Schubert, and Marius Grundmann 


\title{
Demonstration of an ultraviolet ZnO-based optically pumped third order distributed feedback laser
}

\author{
Daniel Hofstetter, ${ }^{\text {a) }}$ Yargo Bonetti, and Fabrizio R. Giorgetta \\ Institute of Physics, University of Neuchatel, 1 A.-L. Breguet, CH-2000 Neuchatel, Switzerland \\ Abdel-Hamid El-Shaer, Andrey Bakin, and Andreas Waag \\ Institute for Semiconductor Technology, Technical University of Braunschweig, Hans-Sommer-Strasse 66, \\ D-38106 Braunschweig, Germany \\ Rüdiger Schmidt-Grund, Mathias Schubert, ${ }^{\text {b) }}$ and Marius Grundmann \\ Institut für Experimentelle Physik II, University of Leipzig, Linnéstr. 5, D-04103 Leipzig, Germany
}

(Received 13 July 2007; accepted 20 August 2007; published online 12 September 2007)

\begin{abstract}
The authors demonstrate an optically pumped $\mathrm{ZnO}$ distributed feedback laser operating at $383 \mathrm{~nm}$. For a large temperature range between 10 and $270 \mathrm{~K}$, the device lased in a single longitudinal mode. Mode selection was accomplished via a third order diffraction grating, which was dry etched into a $120 \mathrm{~nm}$ thick $\mathrm{Si}_{3} \mathrm{~N}_{4}$ layer deposited on the $\mathrm{ZnO}$ active region. They observed a spectral linewidth of $0.4 \mathrm{~nm}$, a pump threshold intensity of $0.12 \mathrm{MW} / \mathrm{cm}^{2}$, and a peak output power of $14 \mathrm{~mW}$. From wavelength versus temperature measurements, they deduced a temperature tuning coefficient of the $\mathrm{ZnO}$ refractive index of $9 \times 10^{-5} \mathrm{~K}^{-1}$. (C) 2007 American Institute of Physics.
\end{abstract}

[DOI: $10.1063 / 1.2783965]$

Starting about ten years ago, $\mathrm{ZnO}$ has become subject to very intense research, mainly in the areas of ultraviolet light emission and optically pumped lasing. Nowadays, there exists a variety of reports on ultraviolet lasing achieved with different experimental configurations. Besides an early report on an e-beam pumped laser, ${ }^{1}$ there are claims of vertical laser emission from a several micrometer thick $\mathrm{ZnO}$ bulk layer, ${ }^{2}$ lasing from a disk in whispering gallery modes, ${ }^{3}$ lasing from a $\mathrm{ZnO}$ photonic crystal, and finally "random lasing" from various nanostructured materials such as nanoneedles, ${ }^{5}$ semiconductor powders, ${ }^{6}$ nanocrystallites, ${ }^{7}$ and nanowires. ${ }^{8}$ Although all of the above reports show, at least under certain pump conditions, a more or less pronounced pump intensity threshold accompanied by a certain linewidth narrowing, they lack proof of an unambiguous and simple relation between an independently measurable cavity property and the emission wavelength. This is also well reflected by the fact that in none of the cited reports, the influence of-for instance-the temperature on the emission wavelength was investigated. In this letter, we therefore try closing this gap by the fabrication of a third order distributed feedback (DFB) laser and by establishing an easily verifiable relation between its temperature dependent emission wavelength and the effective refractive index of its planar waveguide. Additionally, we are convinced that instead of random lasing or "Anderson localization,", effects such as superluminescence and high material gain dominate the very good luminescence properties of our $\mathrm{ZnO}$ films.

Fabrication of the layer relied on molecular beam epitaxy using metallic $\mathrm{Zn}$ and $\mathrm{Mg}$ sources and using $\mathrm{H}_{2} \mathrm{O}_{2}$ as an oxidant. Growth started with a thin $\mathrm{MgO}$ nucleation layer on a C-face sapphire substrate and was followed by a $\mathrm{ZnO}$ active layer with a nominal thickness of $200 \mathrm{~nm}$. Both optical and atomic force microscope inspections revealed a smooth

\footnotetext{
${ }^{\text {a)} E l e c t r o n i c ~ m a i l: ~ d a n i e l . h o f s t e t t e r @ u n i n e . c h ~}$

${ }^{b)}$ Present address: Nebraska Center for Materials and Nanoscience, University of Nebraska-Lincoln, Lincoln, NE 68588-0511, USA.
}

surface with a state-of-the-art defect density on the order of $10^{8} \mathrm{~cm}^{-2}$ and a root mean squared surface roughness of $0.2 \mathrm{~nm}^{9}{ }^{9}$ In order to have correct optical parameters at hand, a wavelength dependent ellipsometric characterization was performed on this layer. Detailed information on the experimental procedure and the model analysis of the ellipsometric data can be found in Ref. 10. Spectra of the refractive and the absorption indices for light polarization perpendicular to the wurtzite-structure $c$ axis are presented in Fig. 1. Multiple excitonic features can be recognized, with the lowest peak located at $3.31 \mathrm{eV}$ in the spectra of the absorption index. A total epitaxial layer thickness of $195 \mathrm{~nm}$ was found. Next, we polished a $1 \mathrm{~mm}$ wide bar with two parallel mirrors and mounted the sample on a copper platelet. Optical pumping with a $337.1 \mathrm{~nm} \mathrm{~N}_{2}$ laser (Laser Science, Inc., VSL-337i) was performed in a liquid He flow cryostat and in standard vertical pump incidence. The peak emission wavelength as a function of device temperature is shown in Fig. 2. The linewidth remained constant at $3.5-4 \mathrm{~nm}$ which is clearly too

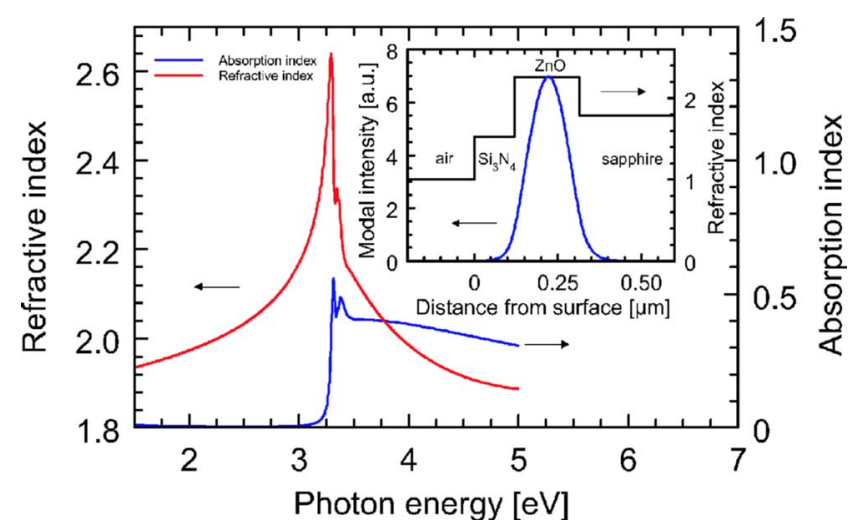

FIG. 1. (Color online) Refractive and absorption indices vs photon energy for light polarization perpendicular to the wurtzite-structure $c$ axis of the $\mathrm{ZnO}$ layer. The inset shows a waveguide simulation based on measured thicknesses and refractive indices of the involved layers. 


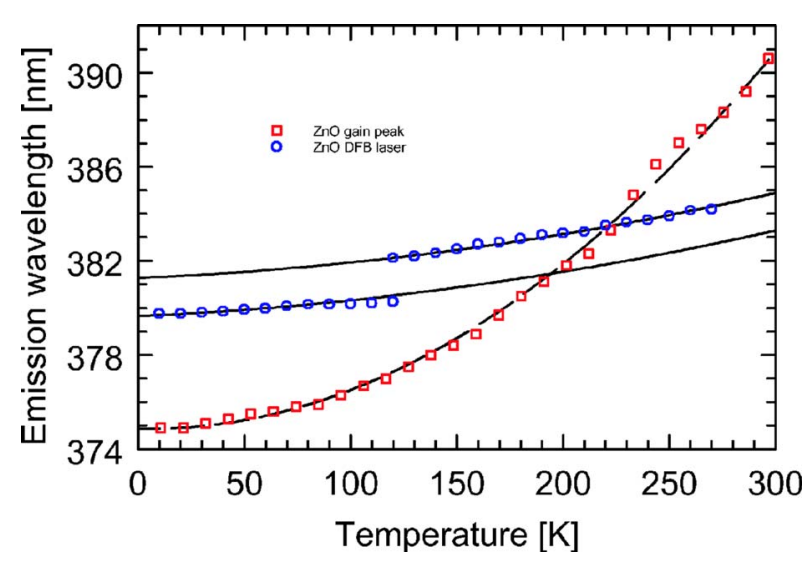

FIG. 2. (Color online) Emission wavelengths as a function of device temperature for the DFB emission (circles) and the luminescence (squares). The lines are guides to the eye.

wide for lasing. Although under inspection by the naked eye, the emission looked slightly speckled, no convincing sign of lasing was observed in the entire temperature range from 10 to $350 \mathrm{~K}$. It is also interesting to note that the luminescence shows only a very small temperature shift between $10 \mathrm{~K}$ and roughly $50 \mathrm{~K}$, whereas the shift at room temperature is much larger. This observation agrees perfectly with recent band gap measurements on similar $\mathrm{ZnO}$ layers. ${ }^{11}$

Since etching of a short-period diffraction grating into $\mathrm{ZnO}$ would be rather difficult, we decided to fabricate the grating in a $120 \mathrm{~nm}$ thick $\mathrm{Si}_{3} \mathrm{~N}_{4}$ layer deposited by plasmaenhanced chemical vapor deposition directly on top of the $\mathrm{ZnO}$. Assuming a line-to-space ratio of 1:1, we then modeled the vertical waveguide of the resulting sapphire/ $\mathrm{ZnO} / \mathrm{Si}_{3} \mathrm{~N}_{4} /$ air sandwich using a commercial waveguide solver. At room temperature, we based ourselves on a target emission wavelength of $\lambda_{0}=390 \mathrm{~nm}$ (taken from Fig. 2), $n_{\text {sapphire }}=1.7880, \quad n_{\mathrm{ZnO}}=2.2974 \quad$ (from Fig. 1), $n_{\mathrm{Si}_{3} \mathrm{~N}_{4} / \text { air }}$ $=(2.075+1) / 2=1.5375,{ }^{12} \quad d_{\mathrm{Si}_{3} \mathrm{~N}_{4}}=120 \mathrm{~nm}, \quad$ and $d_{\mathrm{ZnO}}$ $=195 \mathrm{~nm}$. For these optical parameters, an effective index of $n_{\text {eff }}=2.1851$ was computed. The resulting optical mode along with the corresponding refractive index profile is shown as an inset of Fig. 1. Using the coupled mode theory developed by Kogelnik and Shank, we then calculated an $m$ equal to third order grating period of $\Lambda=\left(m \lambda_{0}\right) /\left(2 n_{\text {eff }}\right)=270 \mathrm{~nm} .{ }^{13}$ However, in order to have somewhat more flexibility in the temperature dependent spectral characterization, a grating with a slightly shorter period of $\Lambda=268 \mathrm{~nm}$ was defined. By simulation of the waveguide effective index with and without the grating layer, we could estimate the coupling constant of the diffraction grating. Using the approximation $\kappa$ $=\left(\Delta n_{\mathrm{eff}} \pi\right) /\left(2 \lambda_{0}\right)$, we found a value of $\kappa=1100 \mathrm{~cm}^{-1}$ in first order, which corresponds to roughly $\kappa=400 \mathrm{~cm}^{-1}$ in third order. Holographic exposure using a $\mathrm{Kr}^{+}$ion laser followed by a $\mathrm{CHF}_{3}$-based reactive ion etching through the entire $\mathrm{Si}_{3} \mathrm{~N}_{4}$ layer completed grating fabrication. The grating period was verified by measuring the diffraction angle of an $\mathrm{Ar}^{+}$ion laser beam. Scanning electron microscopy characterizations confirmed both correct depth and rectangular profile of the grating [see Fig. 3(b)].

The experimental configuration for DFB laser characterization is shown schematically in Fig. 3(a). Optical pumping with a $337.1 \mathrm{~nm} \mathrm{~N}$ laser was performed under an angle of $70^{\circ}$ to the surface normal and in the center of the wafer. The fact of being far away from the wafer edge prevents the light

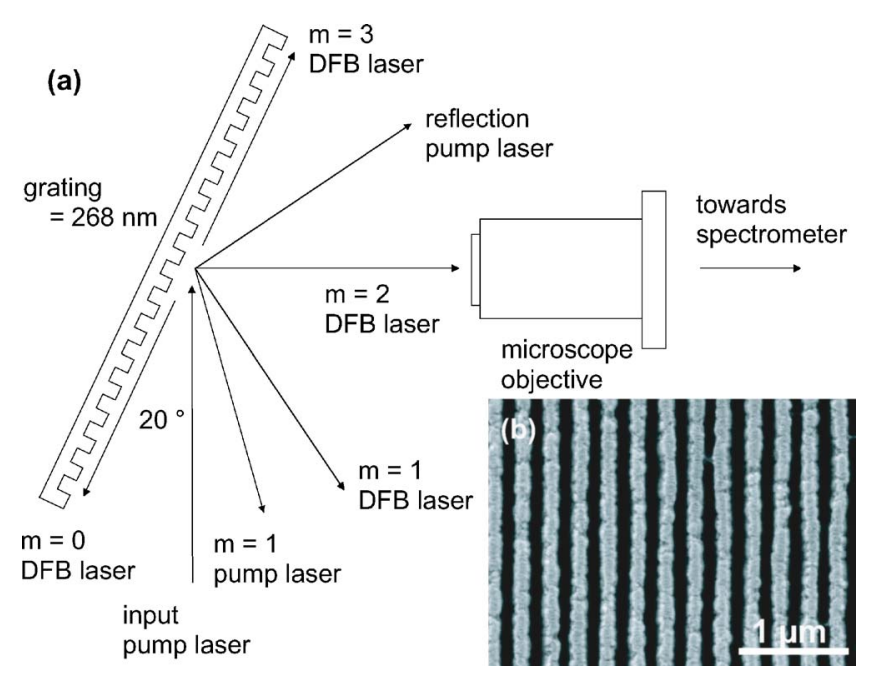

FIG. 3. (Color online) (a) Schematic representation of the optical pump experiment and relevant diffraction orders $m$ of both $\mathrm{N}_{2}$ and ZnO DFB lasers. (b) Scanning electron microscopy image of the diffraction grating.

from seeing facet mirrors which might result in undesired Fabry-Perot lasing. Since a third order grating offers not only feedback in the wafer plane but also two additional surfaceemitting diffraction orders at angles of $\pm 20^{\circ}$ to the surface normal, the output from one of these emissions could be directed on a microscope objective and focused into the $20 \mu \mathrm{m}$ wide entrance slit of a $460 \mathrm{~mm}$ grating spectrometer (Jobin Yvon HR460). The reasons for this particular geometry are better protection of the spectrometer's Si-diode array against dierct $\mathrm{N}_{2}$ laser illumination and geometrical restrictions in our cryostat. Figure 4 shows a series of emission spectra under changing pump intensity at a temperature of $220 \mathrm{~K}$, where the alignment between the Bragg resonance and the gain maximum is ideal. Attenuation of the pump beam was accomplished by a calibrated adjustable neutral density filter. Both the small redshift of the lasing peak and its broadening at higher pump intensity are consistent with a device being locked to a single longitudinal mode. In addition, their relative amount is exactly the same; therefore the minimally measured linewidth of $0.4 \mathrm{~nm}$ must constitute an intrinsic limit having to do with the size and the shape of the pump beam $\left(0.1 \times 3.6 \mathrm{~mm}^{2}\right)$ and the resulting variation in

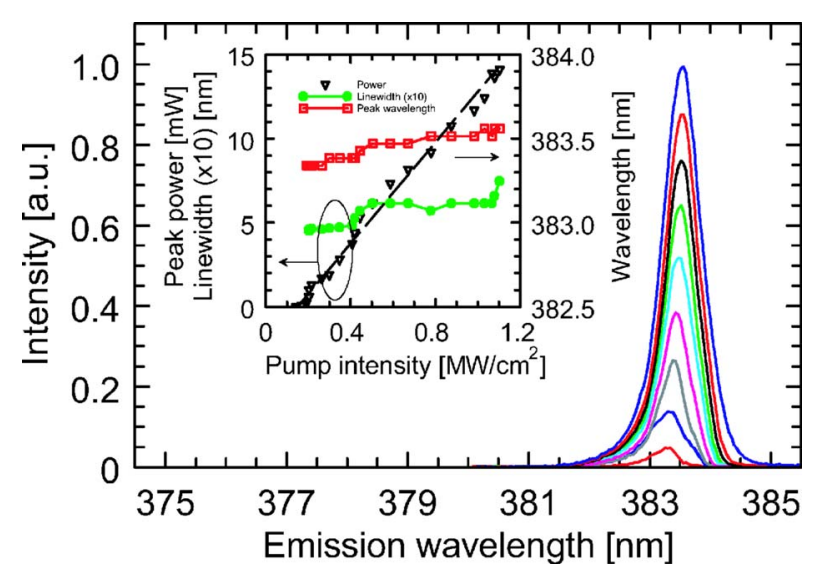

FIG. 4. (Color online) Emission spectra as a function of optical pump power measured at $0.15,0.25,0.35,0.45,0.6,0.8,0.9,1.0$, and $1.1 \mathrm{MW} / \mathrm{cm}^{2}$ (from lowest to highest curve). The inset shows peak power (triangles, left $y$ axis), emission wavelength (squares, right $y$ axis), and linewidth (circles, left $y$ axis, times ten) vs pump intensity curves. 
effective refractive index. In any case, this broadening is due to a small temperature increase at higher pump intensity. Below threshold, a linewidth of $3.5 \mathrm{~nm}$ was seen. In contrast to earlier reports, ${ }^{6}$ but in agreement with the textbook behavior, ${ }^{14}$ we do not see any pump-dependent linewidth narrowing above threshold. In a pulsed laser, thermal chirping will always be considerably larger than any mode selecting process in the cavity; in our example, the $0.3 \mathrm{~nm}$ additional broadening above threshold corresponds to a temperature increase of approximately $25 \mathrm{~K}$.

The inset of Fig. 4 also shows a linear relation between pump intensity and peak output power above threshold. The pump threshold amounts to $0.12 \mathrm{MW} / \mathrm{cm}^{2}$ and the maximum output power peaks at $14 \mathrm{~mW}$. Assuming a p-n-junction voltage drop of roughly $5 \mathrm{~V}$, a future electrically injected laser of comparable performance would have a threshold current density of $\left(0.12 \mathrm{MW} / \mathrm{cm}^{2}\right) / 5 \mathrm{~V}=24 \mathrm{kA} / \mathrm{cm}^{2}$; this is a good value given the large pumped bulk volume. The above threshold pump intensity compares also favorably with the one reported in Ref. $15\left(0.3 \mathrm{MW} / \mathrm{cm}^{2}\right)$ for an optically pumped ultraviolet GaN quantum well laser. The peak power was estimated by comparison to a spectral measurement of a calibrated $\mathrm{GaN} 470 \mathrm{~nm}$ light emitting diode, whereas the pump intensity was estimated via $\mathrm{N}_{2}$ laser peak power, pumped area, and the relevant geometrical factors.

We then investigated the temperature dependence of the emission spectrum. As Fig. 2 shows, the laser remained in the same longitudinal mode between 10 and $120 \mathrm{~K}$ and again between 120 and $270 \mathrm{~K}$. The abrupt wavelength change at $120 \mathrm{~K}$ marks the temperature at which it jumps from the short wavelength to the long wavelength stopband mode. ${ }^{12}$ From the width of this stopband $\left(\Delta \lambda_{\text {Bragg }}=1.6 \mathrm{~nm}\right)$, we were able to compute the coupling constant of the grating via $\kappa$ $=\left(2 n_{\text {eff }} \pi \Delta \lambda_{\text {Bragg }}\right) /\left(\lambda_{0}^{2}\right)$. Thanks to its large depth, high refractive index contrast, and good modal overlap, the grating is very strong $\left(\kappa=700 \mathrm{~cm}^{-1}\right)$, in agreement with our earlier estimation. Furthermore, we performed a series of waveguide simulations at different temperatures in order to characterize the temperature dependence of the $\mathrm{ZnO}$ refractive index. In the temperature region between 120 and $270 \mathrm{~K}$, we found a nearly constant value of $9 \times 10^{-5} \mathrm{~K}^{-1}$ (neglecting the small wavelength change), which agrees very well with recent literature values measured on a $600 \mathrm{~nm}$ thick $\mathrm{ZnO}$ bulk layer. ${ }^{9}$

Figure 5 finally shows three representative examples of the emission spectrum with and without wavelengthselective effect of the diffraction grating. At $120 \mathrm{~K}$, the luminescence is rather far away from the Bragg peak; therefore, the DFB emission is accompanied by a broad luminescence peak on the short wavelength side. At $220 \mathrm{~K}$, the alignment between the luminescence and the Bragg reflection of the grating is ideal, we therefore observe stable single mode operation, as already presented in Fig. 4. Finally, at $270 \mathrm{~K}$, the luminescence peak accompanies the DFB lasing peak at its long wavelength side. Because of the faster temperature tuning of the band gap and the gain peak close to room temperature, no lasing could be observed beyond $270 \mathrm{~K}$. It would, however, be straightforward to fabricate a grating with the computed $270 \mathrm{~nm}$ period; in such an experiment, lasing up to room temperature should be possible.

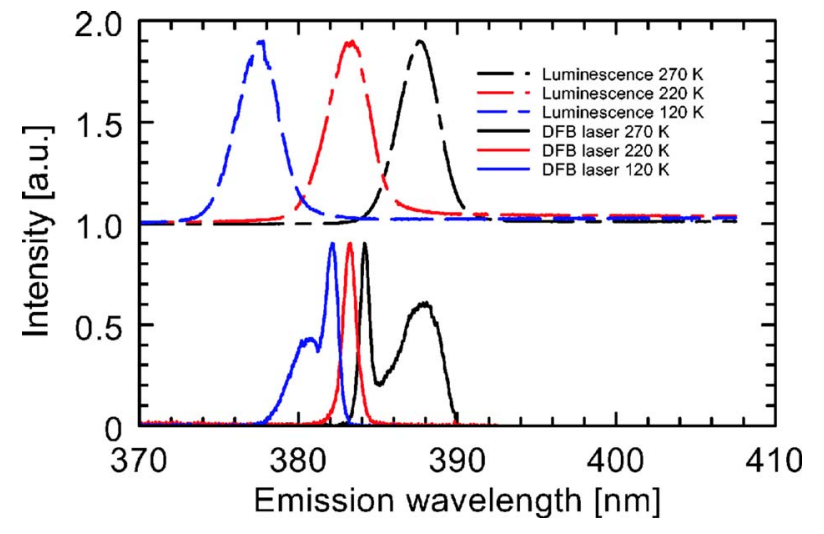

FIG. 5. (Color online) Luminescence (top) and luminescence/DFB laser spectra (bottom) for three representative temperatures (120, 220, and $270 \mathrm{~K}$, from left to right). The wavelength mismatch between the luminescence peaks at $120 \mathrm{~K}$ is due to the higher pump intensity and the concomitant wavelength shift required for DFB lasing.

In conclusion, we have demonstrated a $\mathrm{ZnO}$-based third order DFB laser optically pumped with a $337.1 \mathrm{~nm} \mathrm{~N} \mathrm{~N}_{2}$ laser. Its single mode emission is centered at $383 \mathrm{~nm}$ and remains stable over large temperature ranges within which we verified a smooth refractive index change according to recent literature values. We determined a peak output power on the order of $14 \mathrm{~mW}$.

The authors gratefully acknowledge the financial support of the National Center of Competence in Research "Quantum Photonics" and the Professorship Program of the Swiss National Science Foundation, as well as the Bundesministerium für Bildung und Forschung in Germany.

${ }^{1}$ F. H. Nicoll, Appl. Phys. Lett. 9, 13 (1966).

${ }^{2}$ D. M. Bagnall, Y. F. Chen, Z. Zhu, T. Yao, S. Koyama, M. Y. Shen, and T. Goto, Appl. Phys. Lett. 70, 2230 (1997).

${ }^{3}$ X. Liu, W. Fang, Y. Huang, X. H. Wu, S. T. Ho, H. Cao, and R. P. H. Chang, Appl. Phys. Lett. 84, 2488 (2004).

${ }^{4}$ M. Scharrer, A. Yamilov, X. Wu, H. Cao, and R. P. H. Chang, Appl. Phys. Lett. 88, 201103 (2006)

${ }^{5}$ H. Y. Yang, S. P. Lau, S. F. Yu, A. P. Abiyasa, M. Tanemura, T. Okita, and H. Atano, Appl. Phys. Lett. 89, 011103 (2006).

${ }^{6}$ H. Cao, Y. G. Zhao, S. T. Ho, E. W. Seelig, Q. H. Wang, and R. P. H. Chang, Phys. Rev. Lett. 82, 2278 (1999).

${ }^{7}$ Z. K. Tang, G. K. L. Wong, P. Yu, M. Kawasaki, A. Ohtomo, H. Koinuma, and Y. Segawa, Appl. Phys. Lett. 72, 3270 (1998).

${ }^{8}$ M. H. Huang, S. Mao, H. Feick, H. Q. Yan, Y. Y. Wu, H. Kind, E. Weber, R. Russo, and P. D. Yang, Science 292, 1897 (2001).

${ }^{9}$ A. Bakin, A. El-Shaer, A. Che Mofor, M. Kreye, A. Waag, F. Bertram, J. Christen, M. Heuken, and J. Stoimenos, J. Cryst. Growth 287, 7 (2006).

${ }^{10}$ R. Schmidt, B. Rheinländer, M. Schubert, D. Spemann, T. Butz, J. Lenzner, E. M. Kaidashev, M. Lorenz, A. Rahm, H. C. Semmelhack, and M. Grundmann, Appl. Phys. Lett. 82, 2260 (2003).

${ }^{11}$ S. Heitsch, C. Bundesmann, G. Wagner, G. Zimmermann, A. Rahm, H. Hochmuth, G. Benndorf, H. Schmidt, M. Schubert, M. Lorenz, and M. Grundmann, Thin Solid Films 496, 234 (2006).

${ }^{12}$ Handbook of Optical Constants of Solids II, edited by E. D. Palik (Academic, New York, 1998), 1, p. 771.

${ }^{13}$ H. Kogelnik and C. V. Shank, J. Appl. Phys. 43, 2327 (1972).

${ }^{14}$ D. Hofstetter, L. T. Romano, T. L. Paoli, D. P. Bour, and M. Kneissl, Appl. Phys. Lett. 76, 2337 (2000)

${ }^{15}$ H. Teisseyre, C. Skierbiszewski, A. Khachapuridze, A. FeduniewiczZmuda, M. Skiekacz, B. Łucznik, G. Kamler, M. Kryśko, T. Suski, P. Perlin, I. Grzegory, and S. Porowski, Appl. Phys. Lett. 90, 081104 (2007). 